\title{
Problems of 3D breast imaging
}

by M. Moderhak, A. Nowakowski

Gdansk University of Technology,

Department of Biomedical Engineering

\section{Abstract}

Thermal imaging in Active Dynamic Thermography (ADT) allows analysis of thermal processes forced by external excitation. It seems to be a very promising modality also for medical diagnostics. The procedure of analysis involves thermal models which are described by thermal parameters distributed over a planar surface or inside a 3 dimensional structure. Geometry and thermal parameters determine model thermal properties and are used in simulation of thermal flows. Development of realistic breast thermal models and measurement accuracy for proper determination of heat flow after external excitation are the problems discussed in this paper with the aim of breast cancer diagnostics

\section{Introduction}

In thermal diagnostics usually simple, non-complex models are used. A hemispherical model of the breast [1] used for tumour thermal diagnostics is only a rough approximation of a real breast. There are at least three problems influencing accuracy of thermal analysis. 1) A possibility of creating a precise geometrical model of an organ under test would enable accurate simulation and, as a result, reliable diagnosis. 2) Co-registration of visible and thermal images requires matching the temperature layout to the surface of 3D model of the tested object. 3) Problem of determination of exact heat flow due to limited accuracy of measuring temperature on the surface of a patient. Temperature measurement is strongly affected by 3D geometry of a tested structure and is dependent on the observation angle (1) [2]:

$$
I=f(\cos \Theta)
$$

where $I$ is radiation intensity and $\Theta$ is the angle between normal vector of the examined surface and the direction of observation.

This paper describes a proposal of a 3-dimensional breast imaging method. Figure 1. illustrates registration of stereoscopic breast images by four cameras. Two of them are standard RGB visible-light cameras and the two other are thermal IR cameras. Images registered by the system allow accurate computation of a real 3-dimensional geometry of the breast model. With stereo thermograms it is possible to assign a temperature layout to the surface of the breast model taking into account geometry correction of the emissivity factor.

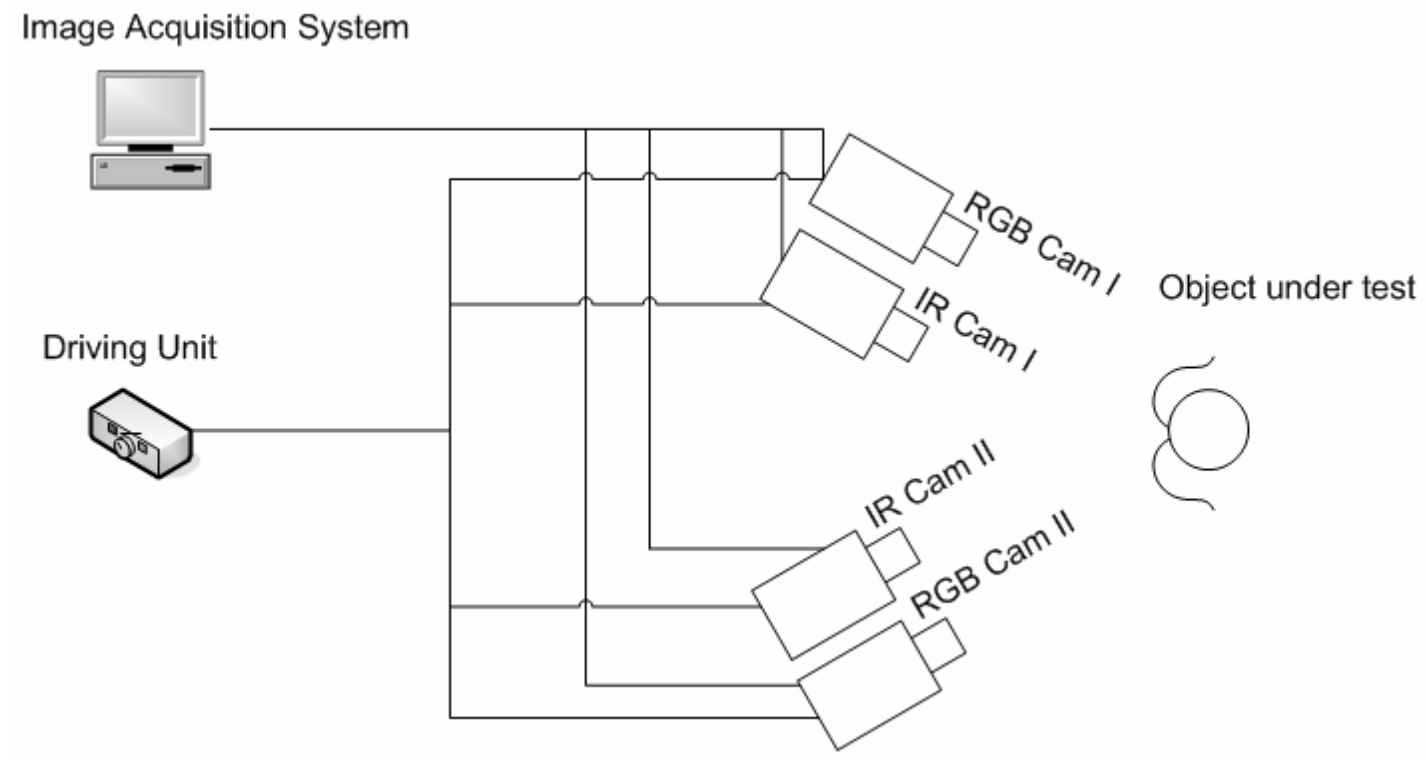

Fig. 1. Configuration of the $3 D$ breast imaging system 


\section{Structure computation and measurement correction}

The procedure of producing such visualization involves inter alia four cameras geometrical calibration, i.e. obtaining their intrinsic parameters such as focal length, skew, aspect ratio, principal point and extrinsic parameters describing the exact camera orientation relative to the world coordinate frame: rotation and translation of each camera. Geometrical calibration is achieved with a special visible light calibration patterns (figure 2 calibration pattern for the RGB camera pair).

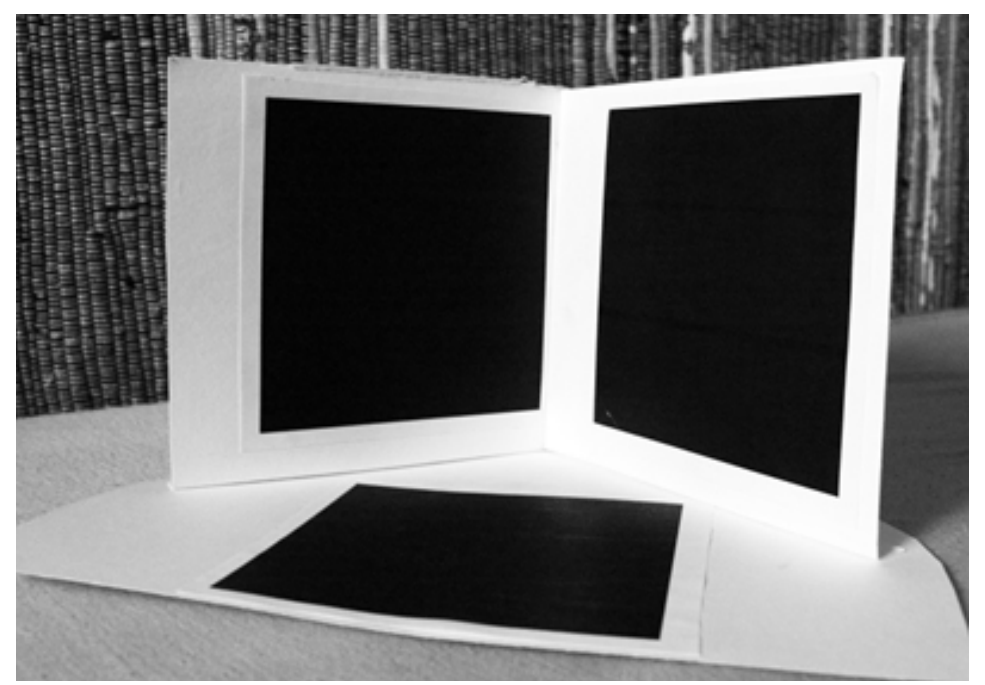

Fig. 2. Calibration pattern for the visible-light camera pair

Formulas (2), (3), (4) and (5) are representing each projection matrix $\mathbf{P}$. $\mathbf{K}$ is a $3 \times 3$ intrinsic parameters matrix, $\mathbf{R}$ is a rotation matrix and $\mathbf{t}$ is a translation. $\mathbf{R}$ and $\mathbf{t}$ are called extrinsic parameters [3]. The camera $I R 1$ is here a reference for the set of four cameras i.e. the external coordinate frame is fixed to its centre.

$$
\begin{aligned}
& P_{I R 1}=K_{I R 1}[I \mid 0] ; \\
& P_{I R 2}=K_{I R 2}\left[R_{I R 2} \mid t_{I R 2}\right] ; \\
& P_{R G B 1}=K_{R G B 1}\left[R_{R G B 1} \mid t_{R G B 1}\right] ; \\
& P_{R G B 2}=K_{R G B 2}\left[R_{R G B 2} \mid t_{R G B 2}\right] .
\end{aligned}
$$

The next step is synthesis of the 3 dimensional model. This part involves identifying pairs of corresponding points in both images. Those correspondences by back projection allow to perform a reconstruction of the registered scene [3]. For 3D visible structure matching of the thermal pattern is the next step. However, the extraction of distinctive features in thermal images is difficult [4]. A method involving video to thermal image matching will be examined. This way thermal and visible light image are fixed to each other for both, right and left pair of cameras. Further on a 3D geometrical model with a temperature layout on its surface is synthesised. Knowing the 3D structure and its relative to camera position it is possible to correct the error resulting from the non zero object observation angle. This allows proper determination of heat flow at the tested breast, after cooling using cryogenic device and further reconstruction of the tested structure.

[1] B. Zhou, W. Shao, G. Wang. The Application of Multi-look in UWB Microwave Imaging for Early Breast Cancer Detection Using Hemispherical Breast Model. Proceedings of the 2005 IEEE EMBC. Shanghai, China, September 1-4, 2005

[2] A. Nowakowski ed. Postępy Termografii - Aplikacje Medyczne, Gdansk 2001.

[3] R. Hartley, A. Zisserman. Multiple View Geometry in Computer Vision. Cambridge University Press (2006).

[4] S. Parkash, P. Y. Lee, T. Caelli. 3D Mapping of Surface Temperature Using Thermal Stereo. ICARV, December 5-8, 2006. Pages $1-4$. 